\title{
Circulating parameters of oxidative stress and hypoxia in normal pregnancy and HELLP syndrome
}

\author{
Ahmet Mentese ${ }^{1, A-D, F}$, Süleyman Güven 2,A,D,F, Selim Demir ${ }^{3, B-D}$, Ayşegül Sümer ${ }^{4, B, C}$, Serap Özer Yamann ${ }^{5, B, C}$ \\ Ahmet Alver ${ }^{5, A, E, F}$, Mehmet Sonmez ${ }^{6, A, E, F}$, Süleyman Caner Karahan ${ }^{5, A, E, F}$ \\ ${ }^{1}$ Program of Medical Laboratory Techniques, Vocational School of Health Sciences, Karadeniz Technical University, Trabzon, Turkey \\ 2 Department of Obstetrics and Gynecology, Faculty of Medicine, Karadeniz Technical University, Trabzon, Turkey \\ ${ }^{3}$ Department of Nutrition and Dietetics, Faculty of Health Sciences, Karadeniz Technical University, Trabzon, Turkey \\ ${ }^{4}$ Department of Nursing, School of Health Services, Recep Tayyip Erdogan University, Rize, Turkey \\ ${ }^{5}$ Department of Medical Biochemistry, Faculty of Medicine, Karadeniz Technical University, Trabzon, Turkey \\ ${ }^{6}$ Department of Hematology, Faculty of Medicine, Karadeniz Technical University, Trabzon, Turkey \\ A - research concept and design; $\mathrm{B}$ - collection and/or assembly of data; $\mathrm{C}$ - data analysis and interpretation; \\ $D$ - writing the article; $E$ - critical revision of the article; $F$ - final approval of the article
}

Address for correspondence

Ahmet Mentese

E-mail: amentese028@gmail.com

Funding sources

None declared

Conflict of interest

None declared

Received on August 2, 2016

Reviewed on January 22, 2017

Accepted on June 9, 2017

DOI

\section{Abstract}

Background. The HELLP syndrome (Hemolysis, Elevated Liver enzymes and Low Platelets) is a complication of severe pre-eclampsia, a condition characterized by oxidative stress elevation caused by disequilibrium between lipid peroxidation and antioxidant defense mechanisms, which, in turn, results in endothelial compromise and free radical-mediated cell damage. While several studies have examined the relationship between pre-eclampsia and oxidative stress, research investigating oxidative and hypoxic status in HELLP syndrome is limited.

Objectives. The aim of this study was to compare the levels of oxidative stress markers - total oxidant status (TOS), total antioxidant status (TAS), oxidative stress index (OSI), and malondialdehyde (MDA) - and a hypoxia marker - carbonic anhydrase IX (CAIX) - in patients with HELLP syndrome and in healthy pregnant women.

Material and methods. A total of 23 women with HELLP syndrome and 30 healthy pregnant women were included in the study. Serum levels of oxidative stress markers were determined using colorimetric methods, while serum levels of CA IX were measured using an enzyme-linked immunosorbent assay (ELISA) kit.

Results. The TOS, OSI, MDA, and CA IX levels were significantly higher in women with HELLP syndrome than in the controls ( $p=0.0001, p=0.0001, p=0.0001$, and $p=0.008$, respectively).

Conclusions. Increased levels of oxidative stress and hypoxia markers in women with HELLP syndrome suggest that oxidative stress and hypoxia may be significantly involved in the pathophysiology of the disease. Further follow-up studies are now needed to investigate the prognostic roles of these parameters in patients with HELLP syndrome.

Key words: oxidative stress, carbonic anhydrases, hypoxia, HELLP syndrome

\subsection{9/acem/74653}

Copyright

Copyright by Author(s)

This is an article distributed under the terms of the

Creative Commons Attribution Non-Commercial License

(http://creativecommons.org/licenses/by-nc-nd/4.0/) 


\section{Introduction}

The HELLP (Hemolysis, Elevated Liver enzymes and Low Platelets) syndrome is a multi-systemic disease with a poor prognosis arising from endothelial dysfunction. It is characterized by multiple organ failure and widespread pregnancy symptoms, such as proteinuria, hypertension and generalized edema, as well as laboratory findings, such as microangiopathic hemolysis, increases in liver function tests and thrombocytopenia. ${ }^{1-4}$ The condition occurs in $0.2-0.6 \%$ of all women during pregnancy and in $10-20 \%$ of cases of eclampsia or pre-eclampsia. The exact mechanism involved in HELLP syndrome, regarded as an extreme form of pre-eclampsia, is uncertain. ${ }^{5}$ The disease occurs as a result of abnormal placental development or function and ischemia, as in severe pre-eclampsia. The $1^{\text {st }}$ stage of the disease is characterized by defective placentation, in which trophoblasts in the decidua prevent complete conversion of the spiral arteries. ${ }^{6}$ This leads to uneven blood perfusion, hypoxia and oxidative stress. Clinical symptoms, such as hypertension, proteinuria and edema, associated with maternal endothelial injury and systemic inflammation, subsequently appear. ${ }^{7}$

Oxidative stress is related to the deterioration of the prooxidant and antioxidant balance. ${ }^{8}$ This results from both excessive reactive oxygen species (ROS) and/or inadequate antioxidant mechanisms. ${ }^{9}$ Oxidative stress increases during uncomplicated pregnancy, due to increased metabolism and a rise in basal oxygen and energy consumption. The placenta, a highly vascular organ, rich in mitochondria and macrophages, is particularly responsible for the production of free radicals. These cell organelles manufacture significant quantities of oxidants, capable of causing injury to the placenta. However, at the same time, antioxidant levels also rise to protect against this injury. Under normal conditions, high levels of oxidants in pregnancy are compensated by antioxidant level elevation, thus averting significant oxidative damage. In contrast to normal pregnancy, when pre-eclampsia occurs, the existing antioxidant levels are incapable of preventing oxidative damage resulting from high levels of free radicals. Additionally, decreases in the levels and activities of a number of antioxidants are observed, including vitamin $\mathrm{E}$, glutathione peroxidase (GPx) and superoxide dismutase (SOD). The combined impact of these factors results in increased lipid peroxidation, endothelial cell dysfunction and vasospasm. ${ }^{10}$ However, the number of studies examining the relationship between oxidative stress and HELLP syndrome is limited. Decreased ferric reducing ability of plasma (FRAP) and increased protein carbonyl levels are reported in women with HELLP syndrome. ${ }^{11}$ Increased levels of oxidative stress markers, such as malondialdehyde (MDA) and proinflammatory cytokines (interleukin-6 and -8) were also shown in infants of pre-eclamptic mothers with HELLP syndrome. ${ }^{12}$ This prospective study of women with HELLP syndrome and normal pregnant women was intended to observe the relative changes in oxidative stress markers (MDA, total oxidant status [TOS], total antioxidant status [TAS], and oxidative stress index [OSI]) and a hypoxia marker (carbonic anhydrase IX [CA IX]).

\section{Material and methods}

\section{Study design and patient settings}

Once the approval had been received from the Karadeniz Technical University Medical Faculty Ethical Committee, Trabzon, Turkey, informed consent was obtained from all subjects. Pregnant women with HELLP syndrome $(\mathrm{n}=23)$ and healthy pregnant women $(\mathrm{n}=30)$ were enrolled as the study and control groups, respectively. Blood samples of the study and control groups were collected during the period January-June 2016. All women in the study group met all the criteria required for the diagnosis of HELLP syndrome: microangiopathic hemolytic anemia with characteristic schistocytes (helmet cells) on blood smear, platelet count (PLT) $\leq 100,000 / \mathrm{mL}$, serum lactate dehydrogenase $(\mathrm{LDH})>600 \mathrm{IU} / \mathrm{L}$, and elevated liver enzymes ( $\geq 70 \mathrm{IU} / \mathrm{L})$ - serum alanine aminotransferase (ALT) and aspartate aminotransferase (AST). ${ }^{13}$ The control group consisted of healthy pregnant women attending the gynecology unit for a routine examination. The exclusion criteria were: a known history of deep vein thrombosis or pulmonary embolism, diabetes mellitus, antiphospholipid syndrome, chronic or acute renal disease, systemic lupus erythematosus, recurrent miscarriage, or thrombophilia; active smoker status; multi-fetal pregnancy; and the use of anticoagulant, non-steroidal anti-inflammatory, corticosteroid, or antiplatelet therapies. The study subjects were not receiving fetal lung maturation or HELLP syndrome treatment steroids at the time of the research.

\section{Ethical approval}

All procedures involving human participants were performed in accordance with the ethical standards of the institutional and/or national research committee and with the 1964 Helsinki Declaration and its later amendments, or comparable ethical standards.

\section{Blood samples and laboratory methods}

Five-milliliter blood samples from each subject were collected in separator gel containing Vacutainer tubes. After clotting, these were centrifuged at $1800 \times \mathrm{g}$ for $10 \mathrm{~min}$. Serum samples were stored at $-80^{\circ} \mathrm{C}$ until the analysis of all parameters. All routine biochemical parameter levels were determined using a Beckman Coulter autoanalyzer (Beckman Coulter, Brea, USA).

The concentrations of CA IX were measured using an enzyme-linked immunosorbent assay (ELISA) kit (R\&D 
Systems, Minneapolis, USA) according to the manufacturer's instructions. The absorbance of samples was measured at $450 \mathrm{~nm}$, using a microplate reader (Molecular Devices, San Jose, USA). The results were expressed as $\mathrm{pg} / \mathrm{mL}$. The minimum detectable level of human CA IX is typically $<2.3 \mathrm{pg} / \mathrm{mL}$.

The MDA concentrations were determined using the method described by Yagi. ${ }^{14}$ Tetramethoxypropane was used as a standard and the MDA levels were expressed as nmol $/ \mathrm{mL}$.

The levels of serum TOS and TAS were determined using a commercial colorimetric kit (Rel Assay Diagnostics, Gaziantep, Turkey) according to the manufacturer's instructions. The TOS results were expressed as $\mu \mathrm{mol}_{2} \mathrm{O}_{2}$ equivalent/L and the TAS results as mmol trolox equivalent/L.

The TOS/TAS ratio was used as OSI. The units of TAS, expressed as mmol trolox equivalent/L, were converted into $\mu \mathrm{mol}$ trolox equivalent/L and OSI was calculated using the formula ${ }^{15}$ :

OSI $=\left[\left(\right.\right.$ TOS, $\mu$ mol $\mathrm{H}_{2} \mathrm{O}_{2}$ equivalent/L $) /$

/(TAS, $\mu$ mol trolox equivalent/L) $\times 10]$.

\section{Statistical analysis}

Statistical analysis was performed using Statistical Package for Social Sciences (SPSS) software v. 23 (SPSS, Inc., Chicago, USA). The results are expressed as medians within the $25^{\text {th }}$ and $75^{\text {th }}$ percentile interquartile range (IQR). All variables were subjected to descriptive statistical analysis. Normal distribution of the concentrations of all parameters in the 2 groups was investigated using the Kolmogorov-Smirnov test. Differences between the groups were analyzed using the Mann-Whitney U test. The area beneath the receiver operating characteristic (ROC) curves was employed in order to calculate the discriminative power of all parameters in terms of the diagnosis or exclusion of HELLP. These ROC curves were used in the calculation of sensitivity, specificity, negative predictive values (NPV), and positive predictive values (PPV) for HELLP. Statistical significance was set at $\mathrm{p}<0.05$.

\section{Results}

The clinical characteristics of the 2 groups are presented in Table 1. The sociodemographic features of the patients were comparable, and no significant differences were observed between the clinical features in the HELLP and control groups.

Median (IQR) serum CA IX, MDA, TAS, TOS, and OSI levels in the groups are summarized in Table 2. Serum CA IX ( $p=0.008)$, MDA, TOS, and OSI levels ( $p=0.0001$ for all) were significantly higher in women with HELLP syndrome compared to control subjects. However, although

Table 1. Demographic, clinical and laboratory characteristics of the groups

\begin{tabular}{|c|c|c|c|c|}
\hline Clinical characteristics & Normal values & $\begin{array}{l}\text { Control group } \\
\qquad(n=30)\end{array}$ & $\begin{array}{l}\text { Study group } \\
\qquad(n=23)\end{array}$ & $p$-value \\
\hline Age [years] & - & $28(24-32)$ & $30(25-34)$ & 0.301 \\
\hline Gravida & - & $2.00(1.00-3.00)$ & $2.00(1.00-3.00)$ & 0.244 \\
\hline Parity & - & $1.00(0.00-1.00)$ & $1.00(0.00-2.00)$ & 0.491 \\
\hline Gestational day & - & $238(219-258)$ & $222(198-245)$ & 0.123 \\
\hline Hemoglobin [g/dL] & $12-17$ & $11.75(11.0-12.0)$ & $11.7(10.4-13.0)$ & 0.732 \\
\hline PLT [number pf cells/mL] & $150-400 \times 10^{3}$ & $\begin{array}{c}210 \times 10^{3} \\
(181,750-258,250)\end{array}$ & $\begin{array}{c}71.5 \times 10^{3} \\
(42,000-90,000)\end{array}$ & $0.0001^{*}$ \\
\hline LDH [IU/L] & $<248$ & $92.0(69.0-111.0)$ & $1,009(789-1,642)$ & $0.0001^{*}$ \\
\hline ALT [IU/L] & $<40$ & $18.0(15.8-25.0)$ & $125(64-209)$ & $0.0001^{*}$ \\
\hline AST [IU/L] & $<40$ & $23.0(17.8-32.5)$ & $159(87-234)$ & $0.0001^{*}$ \\
\hline
\end{tabular}

PLT - platelet count; LDH - lactate dehydrogenase, ALT - alanine aminotransferase; AST - aspartate aminotransferase; ${ }^{*}$ represents significant results $(p<0.05)$ compared to control group.

Table 2. Median values of oxidative stress and hypoxia markers in the groups

\begin{tabular}{|c|c|c|c|}
\hline Parameters & $\begin{array}{l}\text { Control group } \\
\qquad(\mathrm{n}=30)\end{array}$ & $\begin{array}{l}\text { Study group } \\
\qquad(n=23)\end{array}$ & $p$-value \\
\hline CA IX [pg/mL] & $1.10(0.78-1.39)$ & $1.590(0.948-4.530)$ & $0.008^{*}$ \\
\hline MDA $[\mathrm{nmol} / \mathrm{mL}]$ & $0.111(0.082-0.196)$ & $0.622(0.351-1.30)$ & $0.0001^{*}$ \\
\hline TAS [mmol trolox equivalent/L] & $2.38(2.22-2.60)$ & $2.55(2.30-2.87)$ & 0.062 \\
\hline TOS [ $\mu \mathrm{mol} \mathrm{H}_{2} \mathrm{O}_{2}$ equivalent/L] & $10.10(8.89-12.10)$ & $24.10(13.20-28.10)$ & $0.0001^{*}$ \\
\hline OSI & $0.440(0.365-0.505)$ & $0.854(0.580-1.940)$ & $0.0001^{*}$ \\
\hline
\end{tabular}

CA IX - carbonic anhydrase IX; MDA - malondialdehyde; TAS - total antioxidant status; TOS - total oxidant status; OSI - oxidative stress index; * represents significant results $(p<0.05)$ compared to control group. 
Table 3. The ROC curve analysis of all parameters and their sensitivity, specificity, PPV, and NPV values

\begin{tabular}{|c|c|c|c|c|c|c|c|}
\hline Parameters & $\begin{array}{l}\text { Cut-off } \\
\text { point }\end{array}$ & $\operatorname{AUC}(95 \% \mathrm{Cl})$ & $p$-value & $\begin{array}{c}\text { Sensitivity (\%) } \\
(95 \% \mathrm{Cl})\end{array}$ & $\begin{array}{c}\text { Specificity (\%) } \\
(95 \% \mathrm{Cl})\end{array}$ & $\begin{array}{c}\text { PPV } \\
(95 \% \mathrm{Cl})\end{array}$ & $\begin{array}{c}\text { NPV } \\
(95 \% \mathrm{Cl})\end{array}$ \\
\hline CAIX & $>1.53$ & $0.724(0.585-0.837)$ & 0.003 & $61(32-77)$ & $80(66-93)$ & $70(45-89)$ & $73(55-86)$ \\
\hline MDA & $>0.21$ & $0.924(0.819-0.979)$ & 0.0001 & $96(75-100)$ & $77(59-90)$ & $70(49-87)$ & $96(81-100)$ \\
\hline TAS & $>2.71$ & $0.658(0.516-0.782)$ & 0.051 & $40(19-64)$ & $94(80-99)$ & $80(44-98)$ & $73(57-85)$ \\
\hline TOS & $>16.81$ & $0.878(0.760-0.951)$ & 0.0001 & $70(46-88)$ & $97(85-100)$ & $93(68-100)$ & 85 (70-94) \\
\hline OSI & $>0.62$ & $0.820(0.691-0.911)$ & 0.0001 & $65(42-83)$ & $97(83-99)$ & $93(69-99)$ & $78(62-94)$ \\
\hline $\mathrm{ALT}$ & $>35$ & $0.982(0.900-0.997)$ & 0.0001 & $91(72-99)$ & $93(78-99)$ & $91(72-99)$ & $93(78-99)$ \\
\hline AST & $>36$ & $0.987(0.909-0.996)$ & 0.0001 & $96(78-99)$ & $97(83-99)$ & $96(78-99)$ & $97(83-99)$ \\
\hline PLT & $\leq 162,000$ & $1.000(0.932-1.000)$ & 0.0001 & $100(85-100)$ & $100(88-100)$ & $100(85-100)$ & $100(88-100)$ \\
\hline LDH & $>156$ & $0.970(0.881-0.996)$ & 0.0001 & 96 (78-99) & 97 (83-99) & $96(78-99)$ & 97 (83-99) \\
\hline
\end{tabular}

AUC - area under the curve; $\mathrm{Cl}$ - confidence interval; PPV - positive predictive value; NPV - negative predictive value; ROC - receiver operating characteristic; CA IX - carbonic anhydrase IX; MDA - malondialdehyde; TAS - total antioxidant status; TOS - total oxidant status; OSI - oxidative stress index; ALT - alanine aminotransferase; AST - aspartate aminotransferase; PLT - platelet count; LDH - lactate dehydrogenase.

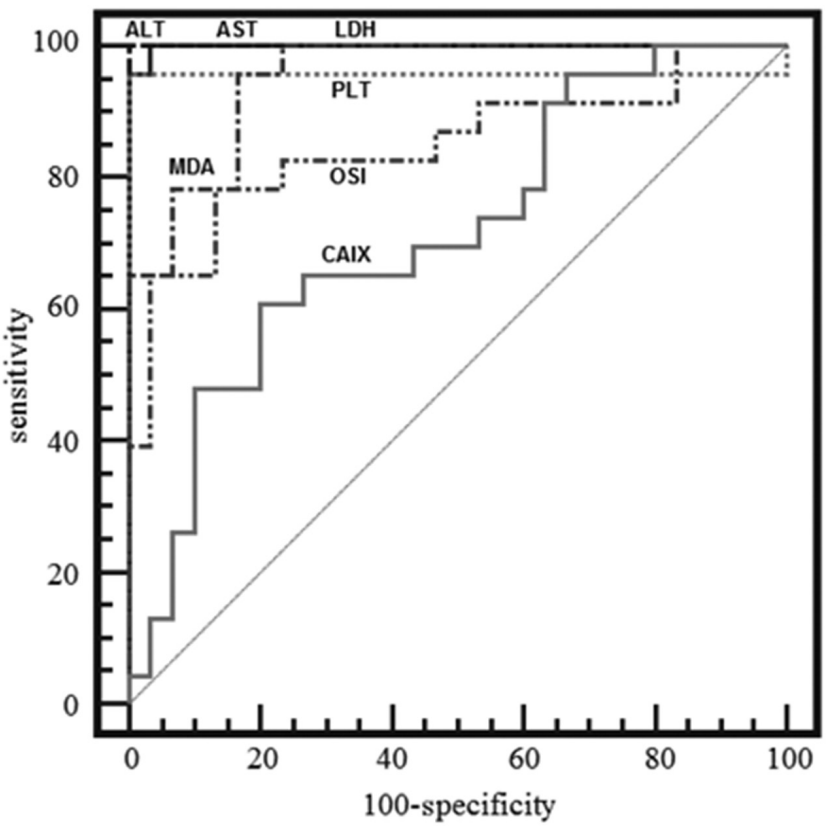

Fig. 1. The ROC curve analyses for all parameters

ROC - receiver operating characteristic; CA IX - carbonic anhydrase IX; MDA - malondialdehyde; OSI - oxidative stress index; ALT - alanine aminotransferase; AST - aspartate aminotransferase; PLT - platelet count; $\mathrm{LDH}$ - lactate dehydrogenase.

serum TAS levels were also higher in the study group compared to the control group, the difference was not statistically significant $(\mathrm{p}>0.05)$.

The ROC curve analysis was also used to quantify serum PLT, ALT, AST, and LDH, and oxidative stress and hypoxia marker levels (Fig. 1). Values for cut-off points, the area under the curve (AUC), sensitivity, specificity, PPV, and NPV for individual parameters are shown in Table 3. In terms of measured oxidative stress and hypoxia parameters, the highest sensitivity was observed in MDA, while the highest specificity was observed in OSI and TOS.

\section{Discussion}

The HELLP syndrome constitutes a group of symptoms observed in pregnant women with either pre-eclampsia or eclampsia, who also exhibit signs of hepatic damage and coagulation anomalies. The disease originates in the placenta, although the pathogenesis is not clearly understood. ${ }^{16}$ Oxidative stress is described as an excess of ROS compared to the buffering capacity of the existing antioxidants. The condition has been implicated in a range of diseases, including atherosclerosis, cancers and preeclampsia. ${ }^{17}$ Good placental oxygenation is essential during pregnancy for cell replication, proliferation and maturation, embryo development, and pregnancy maintenance. In addition, the rises in oxygen concentrations lead to the emergence of oxidative stress markers. As all vascular diseases, pre-eclampsia is characterized by an inflammatory response following ischemia and reperfusion. In the case of pre-eclampsia, placental reperfusion injury leads to a pernicious inflammatory response, implicated in inflammation and oxidative damage mediated by oxidative stress. ${ }^{18}$ Although numerous studies have examined the relationship between pre-eclampsia and oxidative stress, there are only limited studies of oxidative and hypoxic status in HELLP syndrome as an extreme form of pre-eclampsia. ${ }^{11,12,17,19-22}$ The purpose of this study was, therefore, to assess the status of hypoxia and oxidative stress markers in HELLP syndrome. In this study, serum MDA, TOS and OSI levels were significantly higher in patients with HELLP syndrome compared to control groups ( $p=0.0001$ for all). In agreement with our results, Zusterzeel et al. reported decreased levels of FRAP and increased levels of protein carbonyl in women with HELLP syndrome compared to healthy pregnant women. ${ }^{11}$ Mohanty et al. reported higher serum MDA levels in women with pre-eclampsia compared to healthy women. The increase in oxidative stress is further supported by the observation of a negative 
correlation between MDA and antioxidants - vitamin $\mathrm{E}$ and vitamin $\mathrm{C} .{ }^{20}$ Sharma et al. reported increased levels of oxidative stress markers (GPx, SOD and MDA) and decreased levels of antioxidants (vitamin $\mathrm{C}$ and lycopene) in pre-eclamptic women compared to healthy controls. ${ }^{17}$ Chamy et al. reported significantly decreased antioxidant enzyme activity and increased lipid peroxidation in preeclamptic groups compared to healthy pregnant women. ${ }^{19}$ Vanitha Gowda et al. reported that lipid peroxidation and ceruloplasmin oxidase activity were significantly increased in pre-eclampsia compared to normotensive pregnant women. ${ }^{21}$ Additionally, higher MDA and lower SOD levels were reported in women with pre-eclampsia women compared to the control group. ${ }^{22}$ In our study, serum TAS levels were higher in the study group compared to the control group, but this was not statistically significant. We think that this may be attributed to the severity of the disease, the timing of sampling and methodological limitations, such as a relatively small study population.

Carbonic anhydrase IX is a membrane-associated glycoprotein and a member of the $\alpha$-carbonic anhydrase family, responsible for catalyzing the reversible hydration of carbon dioxide to bicarbonate ions and protons. There are 16 known isoforms of CA in humans. ${ }^{23}$ More than 50 genes can be induced by hypoxia, by means of hypoxia inducible factor 1 alpha (HIF-1 $\alpha)$. Carbonic anhydrase IX is a particularly highly inducible and uniformly induced gene. Due to its stability and membrane location, it constitutes a reliable histochemical marker of hypoxia. ${ }^{24}$ There is significant evidence that proves the existence of hypoxia in pre-eclampsia. ${ }^{25} \mathrm{We}$, therefore, determined the CA IX levels as hypoxia markers in this study and observed significantly higher serum CA IX levels in patients with HELLP syndrome compared to healthy controls. Recent studies have shown the importance of $\mathrm{pH}$ in cell death under hypoxia, and the mechanisms of $\mathrm{pH}$ regulation are, therefore, likely to constitute vital pathways for survival. Northern blot analysis has revealed the CA IX expression in tissues, including the heart, liver, hair follicles, articular cartilage, placenta, choroid plexus, and salivary glands. The basal and lateral location of CA IX may similarly imply a role in the transport of bicarbonate or hydrogen ions. Bicarbonate or hydrogen ions in these areas are typically released into the luminal space and these cells may be exposed to extreme $\mathrm{pH}$ values. ${ }^{24}$ Hence, there is a high probability that the activity of this membrane-associated carbonic anhydrase is involved in the preservation of intracellular $\mathrm{pH}$ in HELLP syndrome.

The exact pathobiology of HELLP syndrome is unclear, and no clinical gold standard biomarker for the evaluation of oxidative stress is yet available. ${ }^{5,11}$ Similarly, effective and economical biochemical markers are needed for the early diagnosis of HELLP syndrome. We, therefore, prepared ROC curves for both studied parameters, and gold standards of HELLP syndrome. The results of this analysis are shown in Table 3 and Fig. 1. Although the sensitivity and specificity of the investigated markers were not comparable with those of ALT, AST, PLT, or LDH, we, nevertheless, think that these oxidative stress and hypoxia markers may assist physicians with the diagnosis of HELLP syndrome.

One limitation of our study was its low power. However, to the best of our knowledge, this study is the first to examine CA IX, TAS, TOS, and OSI as biochemical parameters in HELLP syndrome. Further follow-up studies are now needed to investigate the prognostic roles of these parameters in patients with HELLP syndrome.

\section{References}

1. Weinstein L. Syndrome of hemolysis, elevated liver enzymes and low platelet count: A severe consequence of hypertension in pregnancy. Am J Obstet Gynecol. 1982;142(2):159-167.

2. Pokharel SM, Chattopadhyay SK, Jaiswal R, Shakya P. HELLP syndrome: A pregnancy disorder with poor prognosis. Nepal Med Coll J. 2008;10(4):260-263.

3. Basturk M, Tokgoz O, Evsen MS, et al. Emergency approach in HELLP syndrome. J Emerg Med. 2009;8(1):9-12.

4. Benedetto C, Marozio L, Tancredi A, et al. Biochemistry of HELLP syndrome. Adv Clin Chem. 2011;53:85-104.

5. Guven S, Sonmez M, Karahan SC. The role of fibrinolytic and antifibrinolytic activities in the pathophysiology of HELLP syndrome. Hypertens Pregnancy. 2011;30(3):275-286.

6. Brosens JJ, Pijnenborg R, Brosens IA. The myometrial junctional zone spiral arteries in normal and abnormal pregnancies: A review of the literature. Am J Obstet Gynecol. 2002;187(5):1416-1423.

7. Hansson SR, Naav A, Erlandsson L. Oxidative stress in preeclampsia and the role of free fetal hemoglobin. Front Physiol. 2015;5:516.

8. Pisoschi AM, Pop A. The role of antioxidants in the chemistry of oxidative stress: A review. Eur J Med Chem. 2015;97:55-74.

9. Weidinger A, Kozlov AV. Biological activities of reactive oxygen and nitrogen species: Oxidative stress versus signal transduction. Biomolecules. 2015;5(2):472-484.

10. Siddiqui IA, Jaleel A, Tamimi W, Al Kadri HM. Role of oxidative stress in the pathogenesis of preeclampsia. Arch Gynecol Obstet. 2010;282: 469-474.

11. Zusterzeel PL, Rutten $\mathrm{H}$, Roelofs HM, Peters WH, Steegers EA. Protein carbonyls in decidua and placenta of pre-eclamptic women as markers for oxidative stress. Placenta. 2001;22(2-3):213-219.

12. Torrance HL, Krediet TG, Vreman HJ, Visser GH, van Bel F. Oxidative stress and proinflammatory cytokine levels are increased in premature neonates of preeclamptic mothers with HELLP syndrome. Neonatology. 2008;94(2):138-142.

13. Haram K, Svendsen E, Abildgaard U. The HELLP syndrome: Clinical issues and management: A review. BMC Pregnancy Childbirth. 2009;9:8.

14. Yagi K. Lipid peroxides and related radicals in clinical medicine. In: Armstrong D, ed. Free Radicals in Diagnostic Medicine. New York, NY: Plenum Press; 1994:1-15.

15. Turkmen $S$, Mentese $S$, Mentese $A$, et al. The value of signal peptideCUB-EGF domain-containing protein 1 and oxidative stress parameters in the diagnosis of acute mesenteric ischemia. Acad Emerg Med. 2013;20(3):257-264.

16. Glanville T, Walker J. HELLP syndrome. Obstet Gynecol. 2003;5:149-154.

17. Sharma JB, Sharma A, Bahadur A, Vimala N, Satyam A, Mittal S. Oxidative stress markers and antioxidant levels in normal pregnancy and pre-eclampsia. Int J Gynaecol Obstet. 2006;94(1):23-27.

18. Sanchez-Aranguren LC, Prada CE, Riano-Medina CE, Lopez M. Endothelial dysfunction and preeclampsia: Role of oxidative stress. Front Physiol. 2014;5(372):1-11.

19. Chamy VM, Lepe J, Catalan A, Retamal D, Escobar JA, Madrid EM. Oxidative stress is closely related to clinical severity of pre-eclampsia. Biol Res. 2006;39(2):229-236.

20. Mohanty S, Sahu PK, Mandal MK, Mohapatra PC, Panda A. Evaluation of oxidative stress in pregnancy induced hypertension. Indian J Clin Biochem. 2006;21(1):101-105. 
21. Vanitha Gowda MN, Aroor AR, Krishna L. Studies on oxidative stress in preeclampsia. Biomed Res. 2010;21(1):71-79.

22. Khatri M. Circulating biomarkers of oxidative stress in normal pregnancy and preeclampsia and efficacy of antioxidant supplementation. Int J Reprod Contracept Obstet Gynecol. 2013;2(3):304-310.

23. Ambrosio MR, Di Serio C, Danza G, et al. Carbonic anhydrase IX is a marker of hypoxia and correlates with higher Gleason scores and ISUP grading in prostate cancer. Diagn Pathol. 2016;11:45.
24. Potter C, Harris AL. Hypoxia inducible carbonic anhydrase IX, marker of tumour hypoxia, survival pathway and therapy target. Cell Cycle. 2004;3(2):164-167.

25. Tal R. The role of hypoxia and hypoxia-inducible factor- 1 alpha in preeclampsia pathogenesis. Biol Reprod. 2012;87(6):134. 